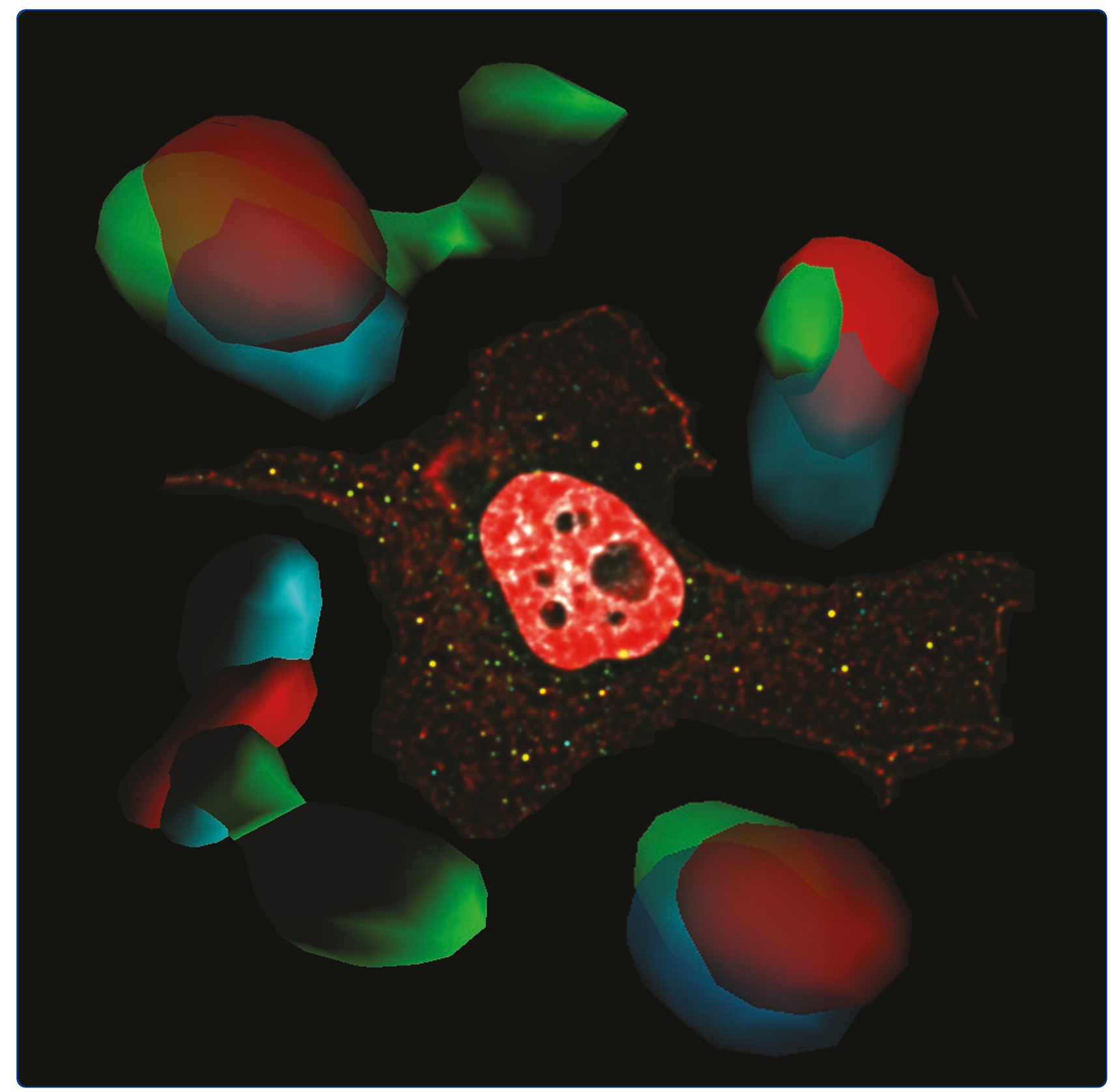

TRIM5a associates with proteasomal subunits in cells while in complex with HIV-1 virions

Lukic et al.

() Biomed Central 


\title{
TRIM5 $\alpha$ associates with proteasomal subunits in cells while in complex with HIV-1 virions
}

\author{
Zana Lukic ${ }^{1}$, Stéphane Hausmann², Sarah Sebastian², Justin Rucci ${ }^{1}$, Jaya Sastri', Seth L Robia ${ }^{3}$, Jeremy Luban ${ }^{2}$ and \\ Edward M Campbell ${ }^{1 *}$
}

\begin{abstract}
Background: The TRIM5 proteins are cellular restriction factors that prevent retroviral infection in a species-specific manner. Multiple experiments indicate that restriction activity requires accessory host factors, including E2-enzymes. To better understand the mechanism of restriction, we conducted yeast-two hybrid screens to identify proteins that bind to two TRIM5 orthologues.

Results: The only CDNAs that scored on repeat testing with both TRIM5 orthologues were the proteasome subunit PSMC2 and ubiquitin. Using co-immunoprecipitation assays, we demonstrated an interaction between TRIM5 $\alpha$ and PSMC2, as well as numerous other proteasome subunits. Fluorescence microscopy revealed co-localization of proteasomes and TRIM5 $\alpha$ cytoplasmic bodies. Forster resonance energy transfer (FRET) analysis indicated that the interaction between TRIM5 and PSMC2 was direct. Previous imaging experiments demonstrated that, when cells are challenged with fluorescently-labeled HIV-1 virions, restrictive TRIM5 $\alpha$ orthologues assemble cytoplasmic bodies around incoming virion particles. Following virus challenge, we observed localization of proteasome subunits to rhTRIM5 $\alpha$ cytoplasmic bodies that contained fluorescently labeled HIV-1 virions.
\end{abstract}

Conclusions: Taken together, the results presented here suggest that localization of the proteasome to TRIM5 $\alpha$ cytoplasmic bodies makes an important contribution to TRIM5 $\alpha$-mediated restriction.

Keywords: TRIM5a, HIV-1, proteasomal subunits, cytoplasmic bodies, immunofluorescence

\section{Background}

The species-specific tropism of numerous retroviruses is determined by host cell proteins, termed restriction factors, which inhibit viral replication at various stages of the viral life cycle. Many members of the TRIM family of proteins act as viral restriction factors. One well-characterized example is the ability of TRIM5 $\alpha$ from rhesus macaques (rhTRIM5 $\alpha$ ) to inhibit human immunodeficiency virus type-1 (HIV-1) [1,2]. TRIM5 $\alpha$ contains RING, B-box, coiled-coil, and B30.2/SPRY domains [3]. The RING domain of TRIM5 $\alpha$ has E3-ubiquitin ligase activity, which is important for restriction and self-ubiquitination. When certain residues within the RING domain are mutated, TRIM $5 \alpha$ loses the ability to potently restrict HIV-1 and self-ubiquitinate, demonstrating the role of ubiquitination

\footnotetext{
* Correspondence: ecampbell@lumc.edu

'Department of Microbiology and Immunology, Stritch School of Medicine, Loyola University Chicago, Maywood IL, USA

Full list of author information is available at the end of the article
}

during TRIM5 $\alpha$ restriction [1]. It is well established that the restriction requires an interaction between the viral capsid lattice and the B30.2/SPRY domain of TRIM5 $\alpha$ [4-6]. Following the binding of the viral core, TRIM5 $\alpha$ mediates an event or series of events that result in the abortive disassembly of the viral core in a manner that prevents the accumulation of reverse transcription (RT) products $[2,7,8]$. Proteasome inhibitors prevent TRIM5 $\alpha$ mediated inhibition of RT products [8,9] and abortive disassembly of the viral core $[10,11]$ without affecting the ability of TRIM5 $\alpha$ to inhibit retroviral infection $[8,9]$. Additionally, TRIM5 $\alpha$ itself is degraded in a proteasome dependent fashion following cytoplasmic delivery of restriction sensitive virus [12].

However, these studies relied on pharmacological inhibitors of proteasome function, which can have pleiotropic effects on the biology of cells. Specifically, proteasome inhibitors such as MG132 deplete the cellular pool of ubiquitin available for cellular processes distinct from
C Biomed Central

(c) 2011 Lukic et al; licensee BioMed Central Ltd. This is an Open Access article distributed under the terms of the Creative Commons Attribution License (http://creativecommons.org/licenses/by/2.0), which permits unrestricted use, distribution, and reproduction in any medium, provided the original work is properly cited. 
degradation. For example, it was recently shown that TRIM5 $\alpha$ can mediate the formation of unanchored K63linked polyubiquitin chains during restriction, which require free cellular ubiquitin and are thought to activate signaling pathways in a manner independent of proteasome activity [13]. In studies relying on proteasomal inhibitors, it is difficult to discriminate between a direct role for proteasomal degradation or an indirect depletion of free cellular ubiquitin that perturbs the generation of K63-linked polyubiquitin chains [13].

Here, we provide evidence for a direct connection between TRIM5 $\alpha$ and the proteasome machinery. The $26 \mathrm{~S}$ proteasome is a barrel shaped, multiprotein complex consisting of a $20 \mathrm{~S}$ core particle (CP) and $19 \mathrm{~S}$ regulatory particle (RP) [14]. The 20S CP is composed of heteroheptameric rings, two outer $\alpha$-rings and two inner $\beta$-rings, each consisting of seven structurally similar $\alpha$ and $\beta$ subunits. The 19S RP contains a base, consisting of RPT and RPN subunits and a regulatory lid comprised of RPN subunits [14]. These subunits were named in studies initially performed using yeast derived proteins, and the vast majority of these names have been maintained in the nomenclature describing mammalian proteasomes. One relevant exception important in this study is the RPT1 subunit, generally referred to as PSMC2. In this study, we use the term PSMC2, though many studies utilize the term RPT1 to describe the mammalian homologue of this protein [14-16]. We observe an interaction between TRIM5 $\alpha$ and the proteasomal subunit PSMC2 using yeast-two-hybrid analysis. This interaction is confirmed using co-immunoprecipitation assays and deconvolution microscopy showing subunits of the proteasome localizing to TRIM5 $\alpha$ cytoplasmic bodies. Using Forster Resonance Energy Transfer (FRET) analysis, we show that TRIM5 $\alpha$ closely associates ( $>5 \mathrm{~nm}$ separation) with proteasomal subunits in cytoplasmic bodies. Finally, we detect the presence of proteasomal subunits in TRIM5 $\alpha$ cytoplasmic bodies associated with restriction sensitive virions.

\section{Results}

TRIM5 $\alpha$ associates with PSMC2 and ubiquitin in a yeasttwo hybrid system

To identify binding proteins of TRIM5, we performed yeast two-hybrid screens using human TRIM $5 \alpha$ and Aotus owl monkey TRIM5Cyp fused to lexA as baits. These baits were screened against a human cDNA library fused to prey. This yielded approximately $3 \times 10^{6}$ transformants for screening. Screening for interaction-positive colonies was performed on selective medium in order to induce the expression of cDNAs in the library. Subsequently, colonies were tested for $\beta$-galactosidase expression and positive clones were subjected to DNA sequencing and BLAST analysis. From this data, proteasomal subunit PSMC2 and ubiquitin were identified as interacting proteins of human
TRIM5 $\alpha$. These hits were individually verified by repeating the two-hybrid system using individual PSMC2 and ubiquitin clones. These clones were found to interact with huTRIM5 $\alpha$ and aotus TRIM5Cyp (Figure 1).

\section{TRIM5 $\alpha$ associates with PSMC2 and other proteasomal subunits by co-immunoprecipitation}

To examine the association between TRIM5 $\alpha$ proteins and proteasomal subunits in human cells, 293T cells were transfected with HA-rhTRIM5 $\alpha$ and FLAG-PSMC2. Following pulldown with anti-HA antibody and blotting with anti-FLAG antibody, we detected FLAG-PSMC2 when HA-rhTRIM5 $\alpha$ was present, but not when pcDNA3.1 vector control was present (Figure 2A), demonstrating an association between PSMC2 and rhTRIM5 $\alpha$. To determine if this association was specific to PSMC2 or other subunits of the proteasome, we, similarly, transfected HA-rhTRIM5 $\alpha$ with FLAG-tagged versions of the proteasomal subunits RPT3, RPT6, and RPN8 (Figure 2B, C, D). All three subunits were specifically pulled down with HA-rhTRIM $5 \alpha$, indicating that rhTRIM5 $\alpha$ associates with numerous subunits of the $26 \mathrm{~S}$ proteasome. To ensure the specificity of this pulldown, we performed a similar pulldown with FLAGtagged Mixed Lineage Kinase 3 (MLK3) [17]. FLAG-MLK3 did not co-immunoprecipitate with HA-rhTRIM5 $\alpha$ (Figure $2 \mathrm{E})$. This demonstrates the specificity of this interaction

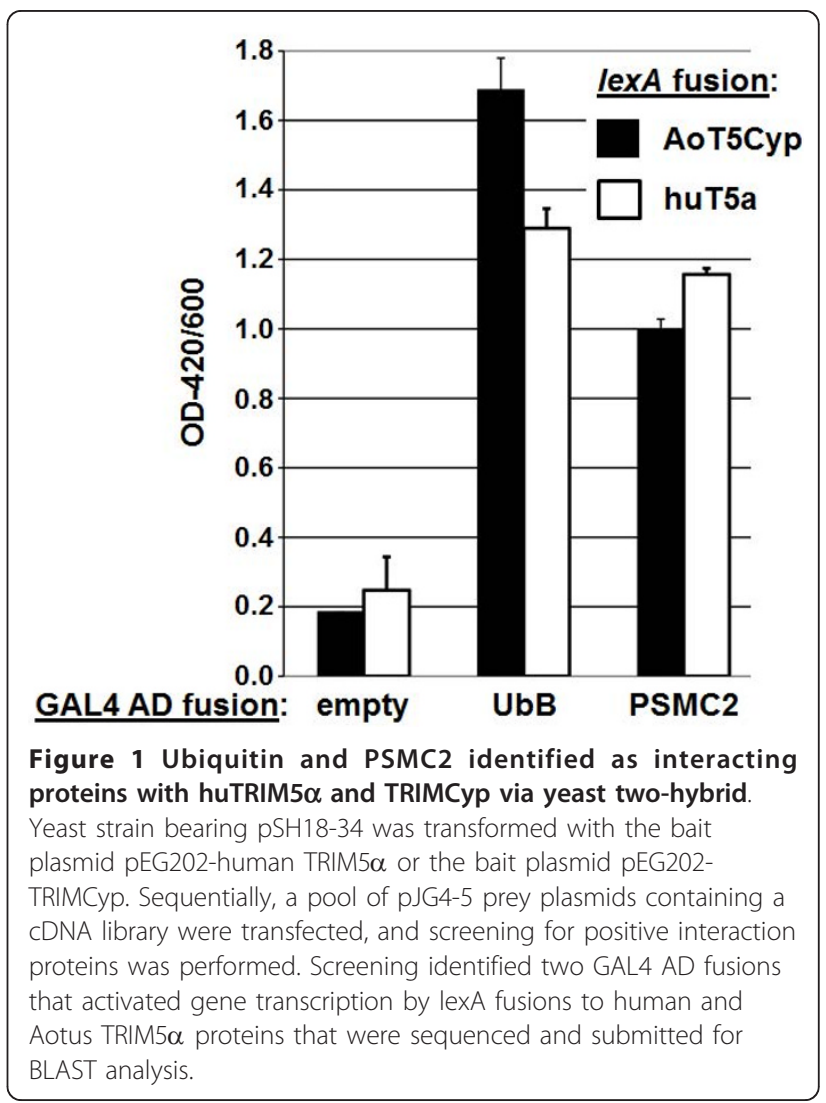




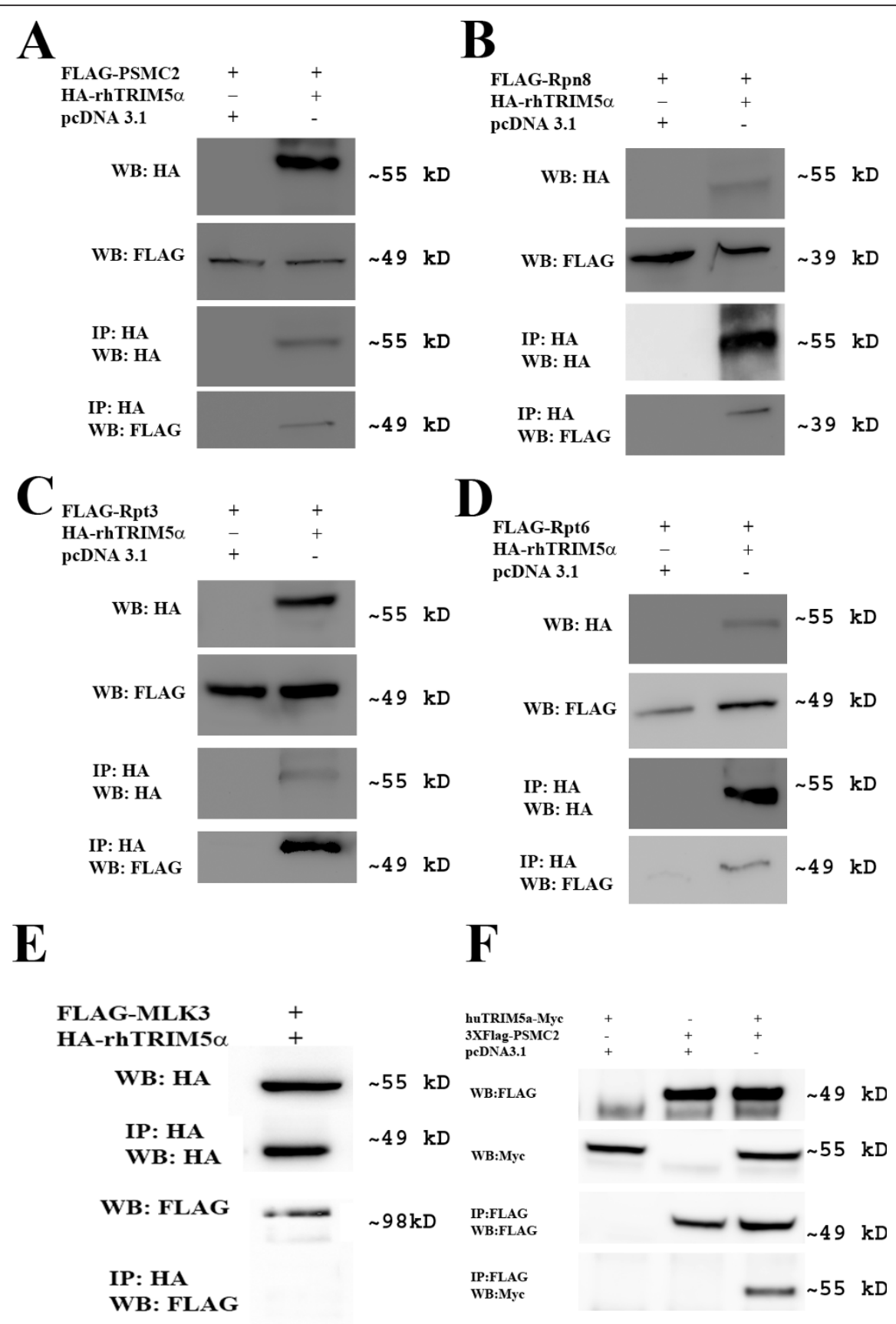

Figure 2 TRIM5 $\alpha$ specifically co-immunoprecipitates with proteasome subunits. (A) Co-immunoprecipitation of PSMC2 with rhTRIM5 $\alpha$. 293T cells were transfected with HA-rhTRIM5 $\alpha$ and FLAG-PSMC2 or pcDNA3.1 empty vector and FLAG-PSMC2 and subjected to immunoprecipitation (IP) with an HA antibody followed by Western blot using indicated antibodies. (B) Co-immunoprecipitation of RPN8 with rhTRIM5 $\alpha$. 293T cells were transfected with HA-rhTRIM5 $\alpha$ and FLAG-RPN8 or pcDNA3.1 empty vector and FLAG-RPN8 and subjected to immunoprecipitation (IP) with an HA antibody followed by Western blot using indicated antibodies. (C) Co-immunoprecipitation of RPT3 with rhTRIM5 $\alpha$. 293T cells were transfected with HA-rhTRIM5 $\alpha$ and FLAG-RPT3 or pCDNA3.1 empty vector and FLAG-RPT3 and subjected to immunoprecipitation (IP) with an HA antibody followed by Western blot using indicated antibodies. (D) Co-immunoprecipitation of RPT6 with rhTRIM5 $\alpha$. 293T cells were transfected with HA-rhTRIM5 $\alpha$ and FLAG-RPT6 or pcDNA3.1 empty vector and FLAG-RPT6 and subjected to immunoprecipitation (IP) with an HA antibody followed by Western blot using indicated antibodies. (E) 293T cells were transfected with HA-MLK3 and subjected to immunoprecipitation with an HA antibody followed by Western Blot. (F) Co-immunoprecipitation of PSMC2 with huTRIM5 $\alpha$. 293T cells were transfected with Myc-rhTRIM5 $\alpha$ and FLAG-PSMC2 or pcDNA3.1 empty vector and FLAG-PSMC2 and subjected to immunoprecipitation (IP) with a FLAG antibody followed by Western blot using indicated antibodies. 
detected by our immunoprecipitation protocol. Additionally, huTRIM5 $\alpha$-Myc was immunoprecipitated with FLAGPSMC2 following transfection in HEK293 cells (Figure 2F), establishing that the association of proteasomal subunits with TRIM5 $\alpha$ is conserved across species.

\section{Proteasomal subunits localize to rhTRIM5 $\alpha$ assemblies in cells}

Next we sought to examine the localization of proteasomal subunits in HeLa cells stably expressing YFP-rhTRIM5 $\alpha$ [18]. Previously, we were unable to detect the localization of rhTRIM5 $\alpha$ and the $20 \mathrm{~S}$ proteasome using a polyclonal antibody [11]. However, the results described above prompted us to speculate that this antibody did not accurately represent the localization of proteasomal subunits by immunofluorescence. We, therefore, initiated a more comprehensive study of proteasome localization using a large panel of antibodies to subunits of the proteasome. As shown in Table 1 and additional file 3, these antibodies typically fell into two categories when utilized for immunofluorescence: those in which a pronounced nuclear localization of the specified subunit was observed and those in which a pronounced nuclear localization was not observed. Numerous reports have shown that proteasome subunits, in addition to maintaining a noticeable and biologically relevant cytoplasmic fraction, exhibit a pronounced nuclear localization [19]. Some antibodies in the panel examined as well as the antibody used in the

Table 1 Characterization of proteasome antibodies for immunofluorescence

\begin{tabular}{|c|c|c|c|}
\hline \multicolumn{4}{|c|}{ Proteasome Antibodies } \\
\hline 19S Base & Catalog Number & Ab Nuclear Localization & Locialization with rhTRIM5a \\
\hline RPT1 mouse & BML-PW8825 & INTERMEDIATE & YES \\
\hline RPT1 rabbit & BML-PW8165 & INTERMEDIATE & YES \\
\hline RPT2 rabbit & BML-PW8305 & NO & NO \\
\hline RPT3 mouse & BML-PW8220 & YES & NO \\
\hline RPT3 rabbit & BML-PW8175 & INTERMEDIATE & YES \\
\hline RPT4 mouse & BML-PW8310 & INTERMEDIATE & NO \\
\hline RPT4 rabbit & BML-PW8830 & NO & NO \\
\hline RPT5 mouse & BML-PW8770 & YES & YES \\
\hline RPT5 rabbit & BML-PW8765 & NO & NO \\
\hline RPT6 rabbit & BML-PW8320 & YES & NO \\
\hline \multicolumn{4}{|l|}{ 20S Alpha } \\
\hline 2 mouse & BML-PW8105 & YES & YES \\
\hline 4 mouse & BML-PW8120 & YES & YES \\
\hline 5 mouse & BML-PW8125 & NO & NO \\
\hline 6 mouse & BML-PW8100 & YES & YES \\
\hline 7 mouse & BML-PW8110 & INTERMEDIATE & NO \\
\hline Core rabbit & BML-PW8155 & YES & YES \\
\hline Alpha $1,2,3,5,6 \& 7$ mouse & BML-PW8195 & NO & NO \\
\hline \multicolumn{4}{|l|}{$20 S$ Beta } \\
\hline 1i (LMP2-13) rabbit & BML-PW8345 & NO & NO \\
\hline 1 mouse & BML-PW8140 & NO & NO \\
\hline 2 mouse & BML-PW8145 & NO & NO \\
\hline 2i (MECL-1) rabbit & BML-PW8350 & YES & NO \\
\hline 3 mouse & BML-PW8130 & INTERMEDIATE & NO \\
\hline 4 rabbit & BML-PW8890 & NO & NO \\
\hline 5i (LMP7) mouse & BML-PW8845 & NO & NO \\
\hline 5i (LMP7) rabbit & BML-PW8355 & YES & NO \\
\hline 5 rabbit & BML-PW8895 & NO & NO \\
\hline 6 rabbit & BML-PW9000 & NO & NO \\
\hline 7 mouse & BML-PW8135 & NO & NO \\
\hline
\end{tabular}

Antibodies were characterized based on their localization in the nucleus. Previous reports have shown that a large pool of proteasomes are located in the nucleus while a fraction remains in the cytoplasm thus the nuclear staining represents antibodies deemed successful for immunofluorescence. 
previous study did not exhibit pronounced nuclear staining (Table 1), casting doubt on the utility of these antibodies for detecting proteasomal subunits by immunofluorescence. In contrast, the majority of antibodies did reveal a pronounced nuclear staining by immunofluorescence. Therefore, we used these antibodies to determine if proteasomal subunits localize to rhTRIM5 $\alpha$ assemblies in HeLa stable cell lines expressing YFP-rhTRIM5 $\alpha$ [18]. Antibodies to numerous subunits demonstrated pronounced accumulation of proteasomal subunits in these assemblies (Figure 3). Specifically, PSMC2 could be detected in these assemblies (Figure 3A). Antibodies to the proteasomal subunits $\alpha 2, \alpha 4, \alpha 6$, and RPT5 also detected a pronounced accumulation of these proteins in YFPrhTRIM5 $\alpha$ assemblies. We also detected proteasomal subunits associated with YFP-rhTRIM5 $\alpha$ cytoplasmic assemblies using a rabbit polyclonal antibody to the $20 \mathrm{~S}$ core particle of the proteasome (Figure 3A). To determine if proteasomal subunit localization was ubiquitous or if localization was specific to a subset of rhTRIM5 $\alpha$ cytoplasmic assemblies, we quantified the proteasome specific immunofluorescent signal associated with individual cytoplasmic assemblies identified by automated image analysis. This analysis revealed that the vast majority of YFP-rhTRIM5 $\alpha$ assemblies contained proteasomal subunits. Using an antibody to the $20 \mathrm{~S}$ core, $99.6 \%$ of subunits had staining levels above background, which was defined as the staining observed using secondary antibodies in the absence of primary antibodies (Figure 3B). Similar results were also observed using mouse monoclonal antibodies to PSMC2 and another base subunit RPT5 as well as $\alpha 4$ and $\alpha 6$ subunits (Figure 3B). Because of the strong degree of $20 \mathrm{~S}$ core staining observed in YFP-rhTRIM5 $\alpha$ assemblies using a rabbit polyclonal antibody, this suggests that virtually all TRIM5 $\alpha$ assemblies associate with proteasomes. Therefore, we believe that alterations in the percentage of cytoplasmic bodies containing individual proteasomal subunits we observed using antibodies represent the ability of these antibodies to reliably detect subunits that are likely present in these assemblies. However, we cannot exclude the possibility that individual subunits are present in more or less abundance, as proteasomal subunit populations may conditionally vary $[20,21]$

\section{FRET analysis reveals a direct association between rhTRIM5 $\alpha$ and proteasomal subunits}

To better understand the association between rhTRIM5 $\alpha$ and proteasomal subunits, we performed immunofluorescence based Forster Resonance Energy Transfer (FRET) analysis on immunofluorescently labeled rhTRIM5 $\alpha$ assemblies and proteasomal subunits. For these studies, we utilized a well-characterized HeLa cell line stably expressing HA-rhTRIM5 $\alpha$ [2]. As previously observed with our YFP-rhTRIM5 $\alpha$ cell line, proteasomal subunits could be observed to localize to rhTRIM5 $\alpha$ assemblies in these cells (Additional File 1). We labeled HA-rhTRIM5 $\alpha$ and proteasomal subunits using secondary antibody combinations that have been previously used to measure FRET interactions in cells [22]. To measure FRET, we utilized the acceptor photobleaching approach, in which the acceptor of a FRET pair is serially photobleached, and the fluorescence of the donor fluorophore is measured over time [23-25]. In this system, if FRET occurs between two fluorophores, then bleaching of the acceptor will result in an increase in the fluorescence of the donor fluorophore as the acceptor fluorophore becomes unable to absorb the energy released from the donor. When PSMC2 was labeled using Alexa546 (donor) and HArhTRIM5 $\alpha$ was labeled using Cy5 (acceptor), serial photobleaching of Cy5 resulted in an increase in the fluorescence detected for PSMC2 (Alexa546). Control bleaching of Cy5 in cells stained with Cy5 secondary antibody alone, in the absence of $\alpha \mathrm{HA}$ primary antibody, did not exhibit this pattern (Figure 4A). Similar results were obtained when a rabbit polyclonal antibody to the $20 \mathrm{~s}$ proteasome was used (Figure 4B). FRET measurement is generally accepted to indicate a direct $(<5 \mathrm{~nm})$ association between two proteins. In this case, because both primary and secondary antibodies were used in this assay, we cannot definitively state this to be true because the addition of a secondary antibody, which has a hydrodynamic radius of $5.5 \mathrm{~nm}$ [26] results in a two-fold decrease in the resolution of this assay $(>10 \mathrm{~nm})$. However, these experiments provide evidence that TRIM5 $\alpha$ and proteasomal subunits exist in very close proximity in rhTRIM5 $\alpha$ assemblies, well below what can be observed using colocalization analysis (Figure 3 ), which is limited by the resolution limit of light microscopy $(\sim 200 \mathrm{~nm})$.

To determine if these proteins were within proximity (typically less than $5 \mathrm{~nm}$ ) and therefore directly interacting, we performed FRET analysis using fusion proteins in which rhTRIM5 $\alpha$ and PSMC2 were fused to the commonly used FRET pair of CFP and YFP, respectively. In live cells transfected with both CFP-rhTRIM5 $\alpha$ and YFPPSMC2, colocalization of rhTRIM5 $\alpha$ and PSMC2 could be readily observed (Additional File 2). We then utilized acceptor photobleaching to determine if FRET occurred between these two proteins in areas of notable colocalization. In this method, the acceptor (YFP-PSMC2) is serially photobleached. FRET is subsequently measured as an increase in donor fluorescence (CFP-rhTRIM5 $\alpha$ ) which occurs as the acceptor is bleached and therefore no longer absorbs the resonant energy from the donor (Additional File 2) [27]. In these experiments, notable increases in the CFP-rhTRIM5 $\alpha$ fluorescence were observed following YFP-PSMC2 photobleaching but an increase was not detected when YFP empty vector was photobleached (Figure 5A). While the FRET difference 


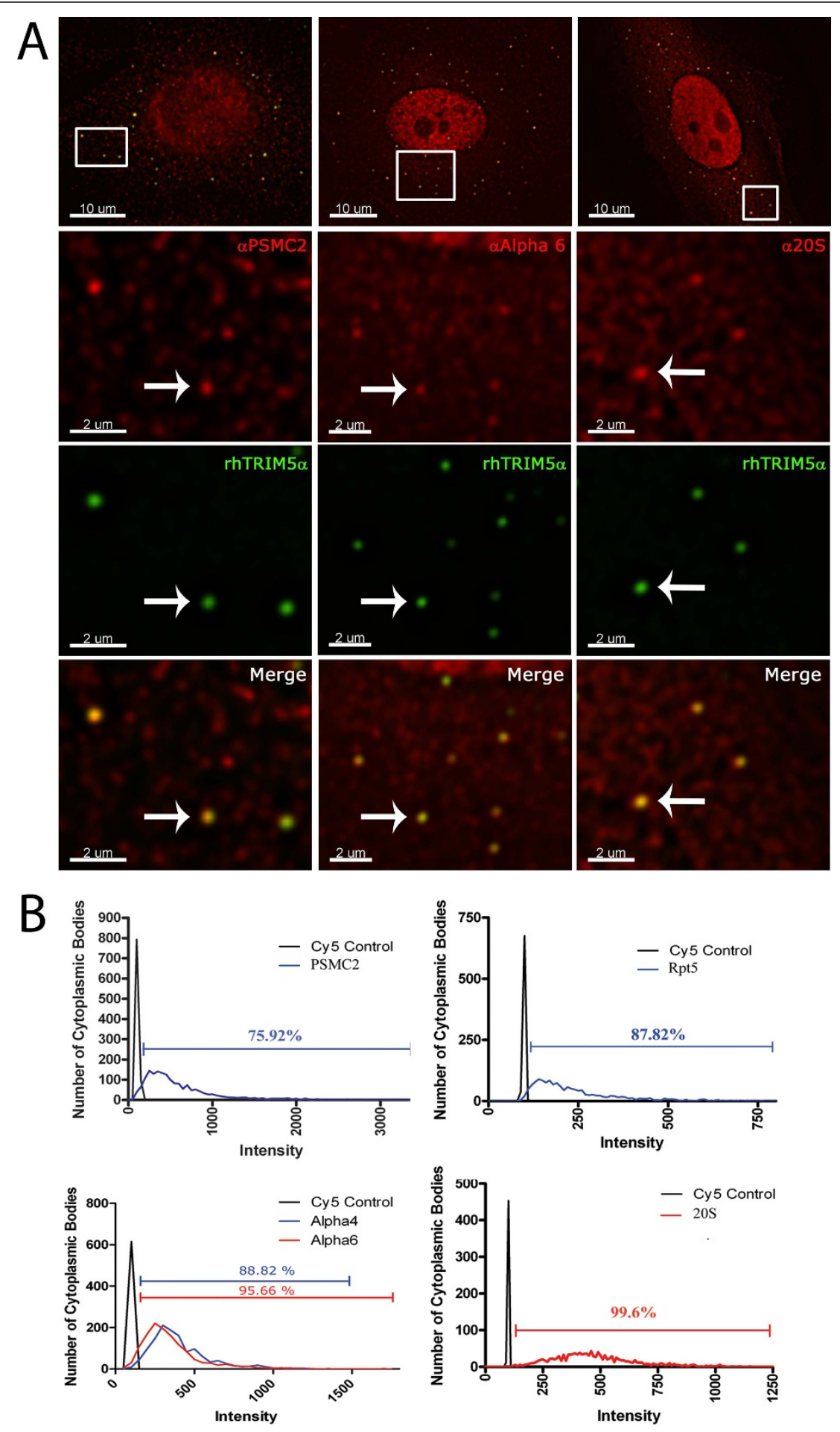

Figure 3 Proteasomal subunits localize to TRIM5 $\alpha$ assemblies at various frequencies. (A) HeLa cells stably expressing YFP-rhTRIM5 $\alpha$ were fixed and immunostained with antibodies specific to the individual subunits of the proteasome. Z-stack images were collected with a DeltaVision microscope equipped with a digital camera using a 1.4-numerical aperture (NA) 100x objective lens, and were deconvolved with SoftWoRx deconvolution software. Individual channel images were superimposed to create the merged panels. (B) Deconvolved images were analyzed for subunit mean fluorescence intensity (MFI) in YFP-rhTRIM5 $\alpha$ cytoplasmic bodies by the use of the Surface Finder function in the Imaris software (Bitplane). For each YFP-rhTRIM5 $\alpha$ cytoplasmic body, the MFI of the subunit was determined and the data was plotted in GraphPad Prism $5^{\circledR}$ software. 


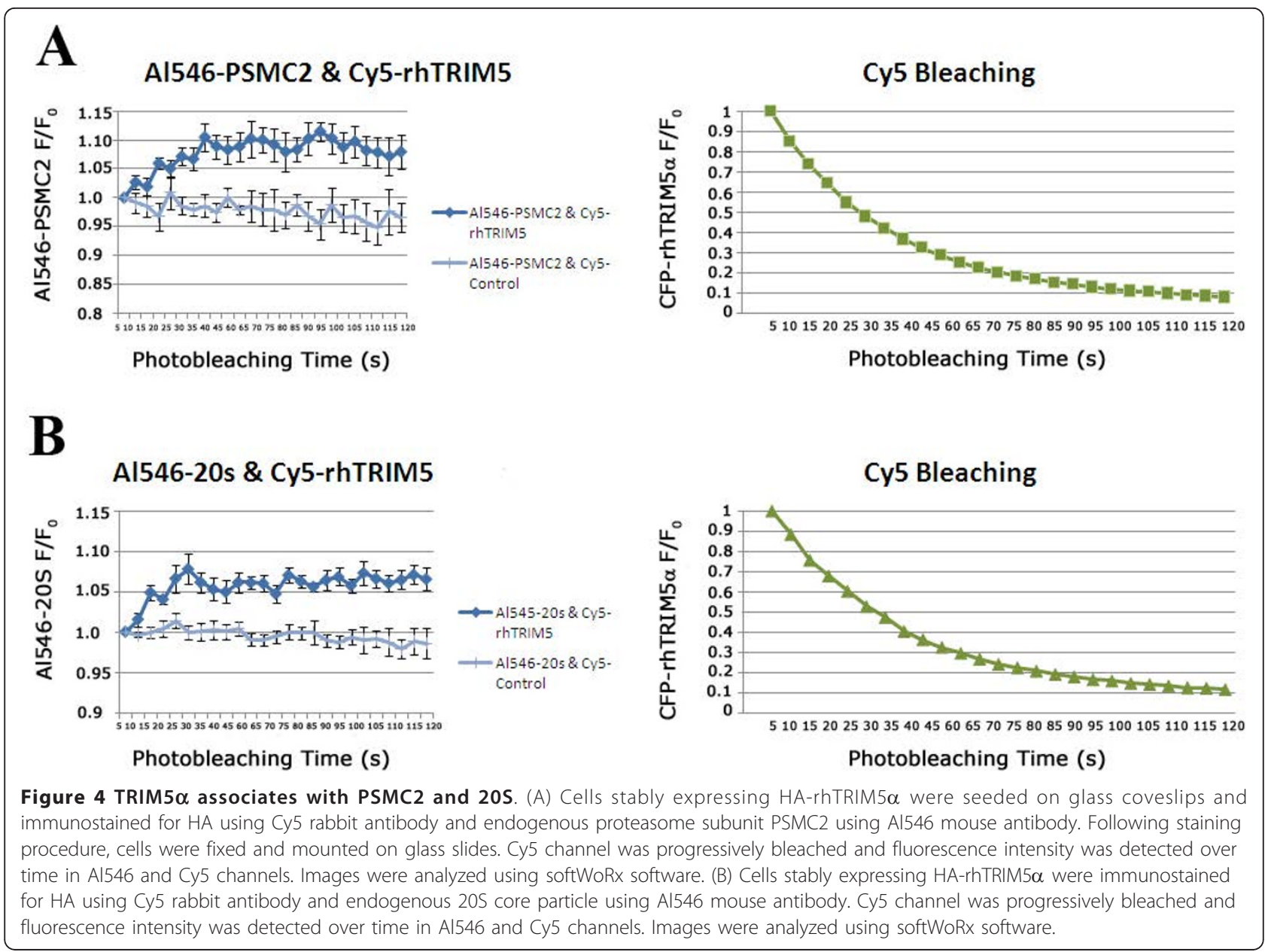

between YFP-PSMC2 and YFP empty vector samples was statistically significant $(\mathrm{p}<0.0001)$, there were also numerous CFP-rhTRIM5 $\alpha$ assemblies in which photobleaching of YFP-PSMC2 did not induce an apparent increase in CFP-rhTRIM5 $\alpha$ fluorescence (data not shown). This suggests that these two proteins do not directly interact in some assemblies. Although we attempted to focus our analysis only on assemblies that did not enter or leave the plane of focus during the course of the experiment, we were concerned that the disparate results obtained were a result of the movement of some assemblies relative to the focal plane during the analysis period. To address this concern, we also performed E-FRET analysis on these assemblies by measuring the amount of YFP fluorescence induced following CFP excitation. Because this method allows a calculation of FRET efficiency derived from three individual images taken in rapid succession, movement of TRIM $5 \alpha$ assemblies during the acquisition period was not a concern. When this method of analysis was utilized, we again observed that the FRET efficiencies of individual assemblies varied considerably (Figure 5B).
It is somewhat surprising that our immunofluorescence based FRET assay consistently exhibited FRET while our fusion protein based assay indicated significant variability in the degree of FRET observed. However, both methods collectively demonstrate that, in some assemblies, a direct interaction between $\operatorname{rhTRIM} 5 \alpha$ and PSMC2 exists.

\section{Proteasomal subunits are present in rhTRIM5 $\alpha$ assemblies} containing HIV-1 virions

The studies above examined the localization of proteasomal subunits to rhTRIM5 $\alpha$ assemblies that exist in cells in the absence of virus. The degree to which pre-existing rhTRIM $5 \alpha$ cytoplasmic bodies resemble assemblies that form around individual virions [11] is unclear. One recent study has found that TRIM $5 \alpha$ forms hexagonal protein assemblies in the presence or absence of in vitro assembled hexameric capsid structures [28], suggesting that TRIM5 $\alpha$ forms structurally similar assemblies in the presence or absence of restriction sensitive virus. The tendency to form such assemblies is enhanced by the presence of these hexameric capsid assemblies [29]. However, 

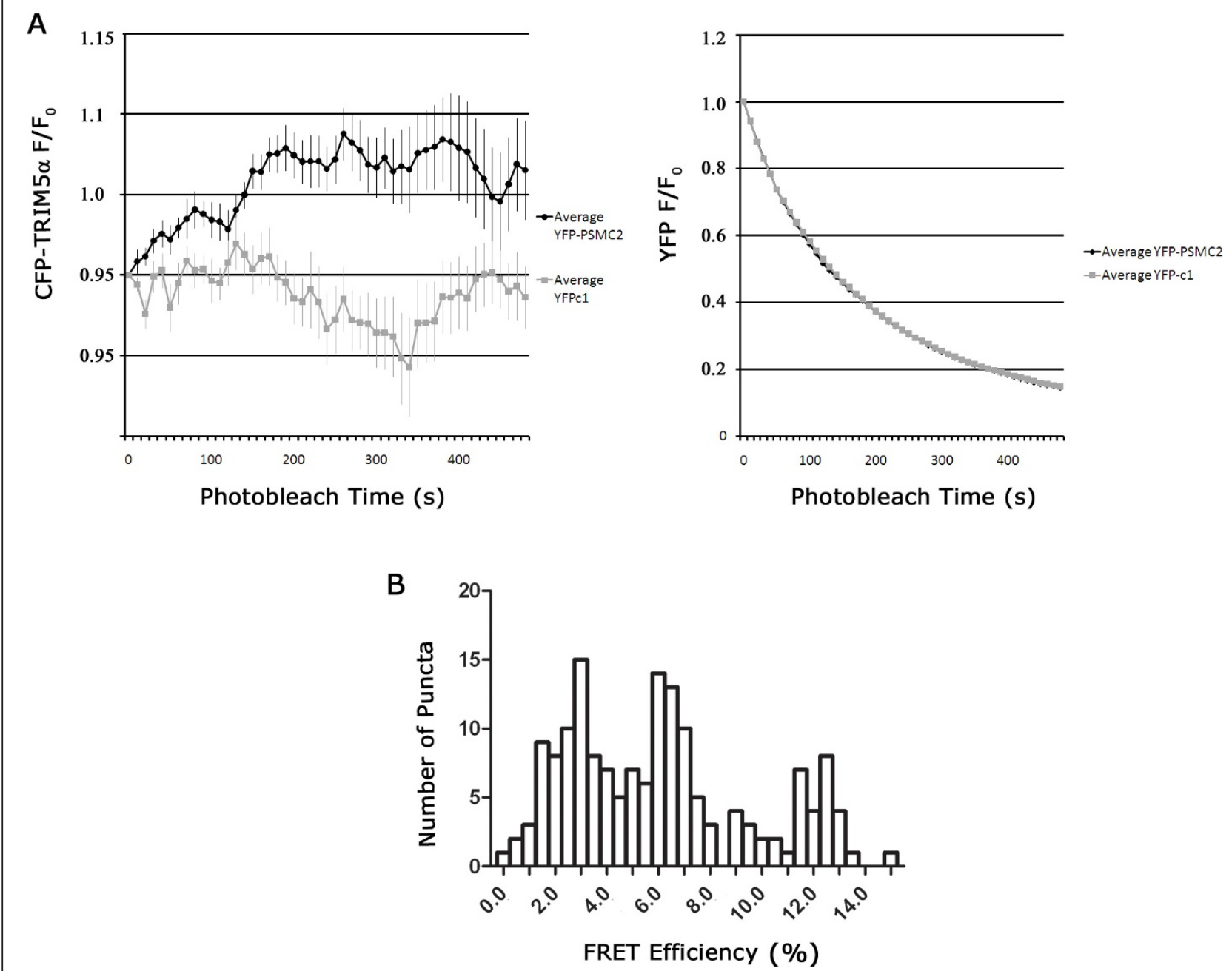

Figure 5 TRIM5 $\alpha$ directly associates with proteasome subunit PSMC2. (A) Progressive acceptor photobleaching of YFP-PSMC2 resulted in an increase in CFP-rhTRIM5 $\alpha$ fluorescence indicating FRET. YFP-PSMC2 or YFP empty vector and CFP-rhTRIM5 $\alpha$ were transfected in HeLa cells at a 1:1 ratio. Over the course of 10 minutes, 50 frames were obtained for YFP and CFP channels. (B) Fluorescence intensities of CFP-rhTRIM5 $\alpha$ and YFP-PSMC2 are recorded. EFRET is calculated as a relative increase of YFP fluorescence intensity (\%) after photobleaching of the CFP FRET acceptor.

there may be biologically important differences between cytoplasmic assemblies of TRIM5 $\alpha$ that form around a restriction sensitive virus and those that form in the absence of virus. We, therefore, sought to determine if TRIM5 $\alpha$ assemblies that form around restriction sensitive virus also contain proteasomal subunits. To this end, we infected a HeLa cell line stably expressing YFP-rhTRIM5 $\alpha$ with low levels of pre-existing cytoplasmic bodies with VSV-g pseduotyped HIV-1 virions fluorescently labeled with an mCherry-Vpr fusion protein $[11,30]$. Following infection for 30 minutes, cells were fixed, stained for proteasomal subunits and quantified for any colocalization between rhTRIM5 $\alpha$ formed cytoplasmic bodies, restriction sensitive virus, and proteasomal subunits (Figure 6). As the engagement of the viral capsid by rhTRIM5 $\alpha$ rapidly leads to the loss of virally associated fluorescent signal, [11], only a small percentage of viral particles could be observed associating with TRIM5 $\alpha$ in fixed cell images in the absence of proteasome inhibitor, as we have previously reported [11]. However, when we did detect such complexes, both proteasomal subunit PSMC2 and the $20 \mathrm{~S}$ core localized to cytoplasmic assemblies of TRIM5 $\alpha$ that formed around a restriction sensitive virus. When these images were quantified, approximately $60 \%$ of TRIM $5 \alpha$ cytoplasmic bodies that contained virus also contained the $20 \mathrm{~S}$ proteasome (data not shown), demonstrating that proteasomal subunits are recruited to TRIM5 $\alpha$ assemblies which associate with HIV-1 viral complexes. 


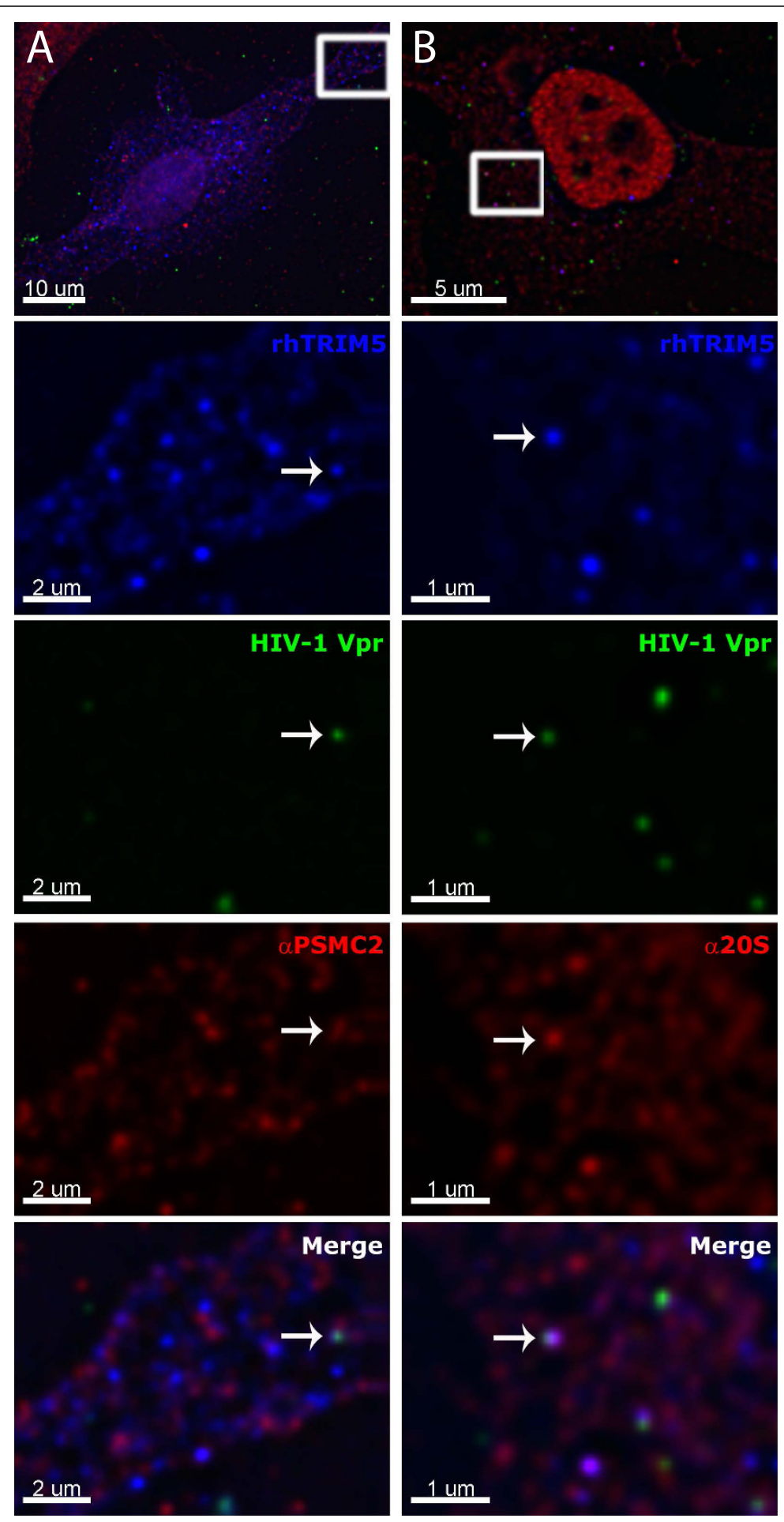

Figure 6 Proteasome associates with TRIM5 $\alpha$ cytoplasmic bodies containing restriction sensitive virus. HeLa cells stably expressing YFPrhTRIM5 $\alpha$ at low levels were seeded on glass coverslips and infected with VSVg pseudotyped HIV-1 virions containing fluorescently tagged mCherry-Vpr for 30 minutes at $37^{\circ}$. Following infection, cells were fixed and stained for the endogenous (A) PSMC2 proteasomal subunits and (B) $20 S$ core. Z-stack images were collected and deconvolved using Deltavision deconvolution software. Individual channel images are superimposed to create the merged panels. 


\section{Discussion}

Previous studies utilizing pharmacological proteasome inhibitors have suggested a role for proteasome mediated degradation in rhTRIM5 $\alpha$ restriction $[8,9,11,12]$. However, evidence demonstrating an actual interaction between rhTRIM5 $\alpha$ and the proteasome has not been published. Due to the potential pleiotropic effects of proteasomal inhibitors on cellular function, such as depletion of the free cellular ubiquitin, it is possible that previously obtained results may not represent a direct role for the proteasome in HIV-1 restriction mediated by TRIM5 $\alpha$. Indeed, recent data have described a proteasome independent role for ubiquitin and rhTRIM5 $\alpha$ E3- ligase activity in retroviral restriction [13]. In this work, we utilized four methods to demonstrate an interaction between proteasomal subunits and rhTRIM5 $\alpha$. Yeast two-hybrid analysis of a cDNA library containing $9.6 \times 10^{6}$ clones identified PSMC2 as a huTRIM5 $\alpha$ and TRIM5Cyp interacting protein. We also used co-immunoprecipitation and immunofluorescence localization studies to show an association between various proteasomal subunits, including PSMC2 and rhTRIM5 $\alpha$. Using FRET analysis, we show a direct interaction between PSMC2 and rhTRIM5 $\alpha$ in cytoplasmic rhTRIM5 $\alpha$ assemblies.

Notably, the majority of studies examining the turnover of TRIM $5 \alpha$ have observed only a mild effect of proteasome inhibitors on TRIM5 $\alpha$ turnover $[8,10,31,32]$ suggesting that the turnover of TRIM $5 \alpha$ in the absence of the virus occurs in a manner that is predominantly proteasome independent. We also observe that the cell lines used in these studies show similar sensitivity to proteasome inhibitors (data not shown). However, one study showed that, following exposure to restriction sensitive virus, TRIM5 $\alpha$ degradation transitions to a proteasome dependent mechanism [12]. However, the mechanism by which TRIM5 $\alpha$ becomes sensitive to proteasomal degradation remains unclear. Our FRET experiments suggest a possible explanation for this observation.

FRET analysis of the interaction between PSMC2 and rhTRIM5 $\alpha$ provides insight into the nature of this association and its underlying biology. We observed an apparent discord between our immunofluorescence based FRET analysis and our fluorescent protein based FRET analysis. There are two possibilities to explain this discrepancy. First, our fluorescent protein based FRET may have yielded variable results because FRET measurements in this assay can be affected if the fluorescent fusion protein does not exhibit the same full biological activity as the native protein. While our previous studies have demonstrated that fluorescent tags do not alter the activity of rhTRIM $5 \alpha$ restriction, it was not feasible to similarly characterize the biological activity of our YFP-PSMC2 construct, which may have contributed to some of the variability observed in these experiments. However, it is also possible that the differences between these assays were due to decreased spatial stringency afforded by the utilization of antibodies used to detect rhTRIM5 $\alpha$ and endogenous PSMC2 in our immunofluorescent FRET. This decreased stringency may be due to: 1) the hydrodynamic radius of the antibody versus the fluorescent protein size or 2) a single donor can transfer energy to multiple acceptors in immunofluorescent FRET while the same phenomenon does not occur in the fluorescent protein based FRET. A single antibody has a hydrodynamic radius of $5.5 \mathrm{~nm}$ [33] while a fluorescent protein is $2-3 \mathrm{~nm}$ in size [34]. Therefore, the use of antibodies may have allowed FRET to occur despite the lack of direct interaction between PSMC2 and rhTRIM5 $\alpha$ in all cases. A possible explanation for the observed heterogeneity using fluorescent protein based FRET is that another protein frequently separates rhTRIM $5 \alpha$ from the proteasome machinery. In our previous work, we showed that p62 directly associates with rhTRIM5 $\alpha$ using fluorescent protein FRET and that p62 siRNA knockdown increases the turnover rate of rhTRIM $5 \alpha$, reducing the steady state expression of TRIM5 $\alpha$ [25]. We, therefore, speculate that the differences noted in our FRET analyses indicate that p62 may reduce the accessibility of rhTRIM5 $\alpha$ to degradation in the absence of virus. In some assemblies, this sequestration of rhTRIM5 $\alpha$ by p62 may be less efficient, allowing the proteasome machinery to engage rhTRIM5 $\alpha$ in these assemblies. This would be consistent with the mild but incomplete sensitivity of TRIM $5 \alpha$ turnover to proteasomal inhibitors $[8,10,31]$

We also show that proteasomal subunits associate with rhTRIM5 $\alpha$ assemblies containing fluorescently labeled HIV-1 virions. We have previously shown that the formation of rhTRIM5 $\alpha$ assemblies around HIV-1 virions leads to the rapid loss of virion fluorescent signal [11]. Taken together with our imaging data presented here, showing proteasomal subunits are present in rhTRIM5 $\alpha / \mathrm{HIV}-1$ complexes, the data suggest that proteasomal subunits are rapidly recruited to these complexes and they are important to the restriction process mediated by TRIM5 $\alpha$. Mechanistically how the proteasome is engaged during the restriction process is not known. We detected proteasomal subunits in a greater percentage of preexisting cytoplasmic bodies (> 90\%) (Figure 3) compared to cytoplasmic bodies containing HIV-1 virions ( $60 \%)$. The simplest explanation for this observation is that proteasomal subunits are recruited to cytoplasmic bodies which form de novo around a restriction sensitive viral core. This would be consistent with other studies which have found that preexisting cytoplasmic bodies are not required for restriction $[35,36]$. The recruitment of proteasomal subunits to this complex, sufficient to be detected by immunofluorescence, 
would then occur. Our fixed cell imaging likely detected complexes in various stages of this process, leading to a reduced number of these assemblies containing detectable amount of proteasomal subunits, compared to preexisting cytoplasmic bodies.

Precisely how the proteasome is involved in restriction is unclear. It is possible that the proteasome recognizes the virus complexed with TRIM5 $\alpha$, leading to direct degradation of viral components. An alternative possibility is that that the proteasome recognizes TRIM5 $\alpha$ directly, which is itself degraded in a manner that leads to the abortive disassembly of the viral core without the direct degradation of viral proteins. The foundation for either possibility has been described here, in which we show a direct role for the proteasome in rhTRIM5 $\alpha$-mediated restriction. In the future, we will pursue experiments to explore either possibility.

\section{Conclusions}

Here, we show that proteasomal subunits associate with TRIM5 $\alpha$ in multiple ways, and this association also occurs while in complex with restriction sensitive HIV-1 virions. These findings suggest that association of the proteasome with TRIM5 $\alpha$ is relevant to TRIM5 biology and restriction.

\section{Materials and methods Yeast Two Hybrid Screen}

The yeast-two-hybrid screen was based on previously described reagents and protocols [37]. Yeast strain EGY48 (MATa trp1 his3 LEU2::pLexAop6-LEU2) bearing pSH18-34, a reporter plasmid containing 8 lexA-binding sites that direct transcription of $l a c Z$, was sequentially transformed with the bait plasmid pEG202-humanTRIM5 $\alpha$, or the bait plasmid pEG202-TRIMCyp. Then yeast was transformed with pJG4-5 (prey), > 90\% of which contain a HeLa cell cDNA library, in a pool of 9.6 $\times 10^{6}$ individual clones. After each transformation, colonies were selected on synthetic drop-out medium. Transformation efficiency for the prey plasmid was $10^{5}$ colonies per $\mu \mathrm{g}$ DNA, and $25 \mu \mathrm{g}$ of the library plasmid pool were used to achieve a total of approximately 2-3 $\times$ $10^{6}$ colonies for screening. Screening for interaction-positive colonies was performed on selective, galactose-containing medium in order to induce the expression of the cDNAs in the library. Subsequently, a filter-lift assay was used to confirm positive clones. Briefly, filters were used to lift colonies from media plates, placed at $-80^{\circ} \mathrm{C}$ for 15 minutes to disrupt yeast cell walls, and incubated on Whatman paper soaked in buffer Z containing X-gal. Colony lifts that turned blue within 30 minutes at $37^{\circ} \mathrm{C}$ were termed putative positive interactors, and the corresponding colonies were picked and analyzed further. Library-cDNA containing plasmids (pJG4-5) were recovered from positive clones. Since these clones contained all three plasmids (reporter, bait, and prey), it was necessary to make use of the KC8 E. coli strain to isolate pJG4-5. KC8 bacteria are trp- and can be selected on TRP- plates that only allow growth if the bacteria contain pJG4-5 (TRP153 selection marker). The library inserts of recovered pJG4-5 plasmids were sequenced, submitted to BLAST analysis, and retested for activity in the twohybrid system as individual clones.

\section{Recombinant DNA Constructs}

HA-tagged rhTRIM5 $\alpha$ and huTRIM $5 \alpha$-Myc have been previously described $[38,39]$. FLAG-tagged proteasomal subunits were generously provided by Dr. Shigeo Murata [40]. 3X-FLAG PSMC2 was generated by PCR-amplification of the PSMC2 open reading frame from pCMV6-ACPSMC2 plasmid (Origene) using primers that introduced unique SpeI and NotI sites at the start codon and 3' of the stop codon, respectively. The PCR product was digested with SpeI and NotI and then inserted into a customized pcDNA3.1 (-)-3XFlag expression vector. This generated PSMC2 was fused to an N-terminal 3X-FLAG. The insert of the resulting pcDNA3.1 (-) 3XF-PSMC2 plasmid was sequenced completely to confirm the presence of the wildtype open reading frame and exclude the acquisition of unwanted mutations during amplification. Yellow fluorescent protein (YFP) C1-PSMC2 was generated by PCR amplifying PSMC2 from a FLAG-PSMC2 plasmid that was generously provided by Dr. Shigeo Murata using the following forward primer 5'-GCGCGAAGCTTGGATG CCGGATT-3' and reverse primer 5'-GCGCGTCGACTCATCAGTTGTATG-3'. The PCR product was inserted into YFP C1 vector (Clontech) using HindIII and SalI (New England Biolabs) restriction sites. The YFP C1PSMC2 clone was confirmed with restriction digest and sequencing analysis to confirm the presence of the wild type open reading frame and fluorescent protein.

\section{Cell culture, transfection and virus production}

HeLa and 293T cells were cultured in complete DMEM containing 10\% fetal bovine serum, penicillin (final concentration $100 \mathrm{U} / \mathrm{ml}$ ), and streptomycin (final concentration $100 \mu \mathrm{g} / \mathrm{ml}$ ). mCherry-Vpr labeled virus was produced by Polyethylenimine (PEI) transfection [41] of 293T cells with $3.3 \mu \mathrm{g}$ of pVSV-G, $5 \mu \mathrm{g}$ of the proviral construct R7 $\Delta$ EnvGFP in which the Nef gene was replaced with GFP and 1.7 ug mCherry-Vpr. Virus was harvested as previously described [42].

\section{Stable cell lines}

HeLa cells stably expressing HA-rhTRIM5 $\alpha$ [43] or YFP-rhTRIM5 $\alpha$ [18] have been previously described. To generate HeLa cells to measure cytoplasmic body formation during viral infection, cells were transduced with 
YFP-rhTRIM5 $\alpha$ retroviral vector and then selected in G418 $(400 \mu \mathrm{g} / \mathrm{mL})$ containing media. Single colony clones were screened by immunofluorescence to identify a cell line that expressed YFP-rhTRIM5 $\alpha$ containing reduced pre-existing cytoplasmic bodies in the absence of restriction sensitive virus.

\section{Antibodies}

Antibodies to various proteasomal subunits were purchased from Enzo Life Sciences (Table 1) and rabbit antiPSMC2 was purchased from Santa-Cruz. Monoclonal Anti-FLAG M2-peroxidase antibody was purchased from Sigma (Catalog Number: A8592), and Anti-HA-peroxidase antibody was purchased from Roche (Catalog Number: 12013819001).

\section{Co-Immunoprecipitation Rhesus TRIM5 $\alpha$ co-immunoprecipitation}

Sub-confluent 293T cells grown in 10-cm dishes were transfected with 10 ug of total plasmid DNA using PEI. 48-hours post transfection cells were washed with $1 \mathrm{ml}$ ice-cold phosphate buffered saline (PBS) and lysed with 1 $\mathrm{ml}$ ice-cold lysis buffer (50 mM Tris, pH 7.4, $125 \mathrm{mM}$ $\mathrm{NaCl}, 1 \% \mathrm{NP}-40)$ supplemented with phosphatase inhibitor cocktail (Roche). Crude cell lysates were collected, transferred to a pre-chilled $2 \mathrm{ml}$ microcentrifuge tube, and solubilized at $4^{\circ}$ for one hour. Following solubilization, cells were sonicated for 10 seconds and centrifuged at $13,000-\times g$ for 20 minutes at $4^{\circ} \mathrm{C}$. $50 \mathrm{uL}$ of supernatant were aliquoted for total cell lysate, and an equal volume of 2X Laemmli sample buffer was added; samples were then boiled for five minutes at $100^{\circ} \mathrm{C}$. To pull down HArhTRIM5 $\alpha$, anti-HA antibody (Sigma) was added to the remaining supernatant at a 1:200 dilution and incubated at $4^{\circ} \mathrm{C}$ for two hours. $50 \mathrm{ul}$ of protein A beads (Miltenyi Biotec) were added to the supernatant and incubated at $4^{\circ} \mathrm{C}$ for an additional hour. Samples were loaded on MACS separation columns (Miltenyi Biotec), followed by three washes with wash buffer (150 mM Tris, pH 7.4, $125 \mathrm{mM}$ $\mathrm{NaCl}$, and 1\% NP-40). Protein complexes were eluted in $30 \mathrm{uL}$ of pre-warmed 1X sample buffer.

\section{Human TRIM5 $\alpha$ co-immunoprecipitation}

Sub-confluent HEK293 cells grown in 10-cm dishes were transfected with $24 \mu \mathrm{g}$ total plasmid DNA using Lipofectamine 2000 (Invitrogen), following the manufacturer's protocol. Forty-two hours post-transfection, cells were washed with $5 \mathrm{ml}$ ice-cold PBS and lysed with $800 \mu \mathrm{l}$ icecold lysis buffer ((50 mM Tris, $\mathrm{pH} 7.5,150 \mathrm{mM} \mathrm{NaCl}, 1 \%$ Triton X-100, 1 mM EDTA 10\% glycerol), supplemented with protease inhibitor cocktail (Roche). Crude cell lysates were collected, transferred to pre-chilled $2 \mathrm{ml}$ microcentrifuge tubes, and centrifuged at $10,000 \times g$ for 10 minutes. The clarified lysate was transferred to pre-chilled microcentrifuge tubes. To prepare antibody conjugated beads, $2 \mu \mathrm{g}$ of antibody (mouse anti-flag M2; Sigma F1804) was conjugated to $50 \mu \mathrm{L}$ of Protein G Dynabeads ${ }^{\circledR}$ (Invitrogen) following the manufacturer's protocol. The beads were washed three times with $1 \mathrm{ml}$ ice-cold lysis buffer, resuspended in $50 \mu \mathrm{L}$ lysis buffer, and added to the clarified cell lysates. After two hours rotating at $4^{\circ} \mathrm{C}$, the bead-immune complexes were washed five times with 1 $\mathrm{ml}$ ice-cold lysis buffer, resuspended in $100 \mu \mathrm{L}$ of $1 \times$ Laemmli sample buffer, incubated at $100^{\circ} \mathrm{C}$ for five min.

\section{Western Blotting}

Samples were loaded into a $10 \%$ polyacrylamide gel for SDS-polyacrylamide gel electrophoresis (SDS-PAGE). After separation, the proteins were transferred to nitrocellulose membrane (Bio-Rad) and detected by incubation with anti-HA conjugated to Horseradish Peroxidase (HRP) (Roche), anti-FLAG (Sigma) and anti-Myc. Secondary antibodies conjugated to HRP (Thermo Scientific) were used where necessary and antibody complexes were detected using SuperSignal ${ }^{\mathrm{TM}}$ West Femto Chemilluminescent Substrate (Thermo Scientific). Chemiluminescence was detected using the UVP EC3 ${ }^{\mathrm{TM}}$ Imaging System (UVP LLC).

\section{Immunofluorescence}

HeLa cells stably expressing YFP-rhTRIM5 $\alpha$ were allowed to adhere to fibronectin-treated glass coverslips and fixed with $3.7 \%$ formaldehyde (Polysciences) in 0.1 M PIPES, pH 6.8 [piperazine- $N, N^{\prime}$-bis(2-ethanesulfonic acid)] (Sigma). Monoclonal and polyclonal primary antibodies raised against various proteasomal subunits were used (Enzo Life Sciences, Table 1). Primary antibodies were secondarily labeled with Cy5 fluorophore-conjugated donkey anti-mouse or anti-rabbit antibody (Jackson ImmunoResearch). Images were collected with a DeltaVision microscope (Applied Precision) equipped with a digital camera (CoolSNAP HQ; Photometrics), using a 1.4-numerical aperture $100 \times$ objective lens, and were deconvolved with SoftWoRx deconvolution software (Applied Precision).

\section{Image Analysis}

20 Z-stack images were acquired using identical acquisition parameters. Surfaces for cytoplasmic bodies in all samples analyzed were defined by using a fluorescence threshold (600 relative fluorescence units) for YFPrhTRIM5 $\alpha$, and all YFP-rhTRIM5 $\alpha$ bodies over a volume of $0.011 \mu^{3}$ were used in the analysis. Deconvolved images were analyzed for PSMC2, RPT5, Alpha 4, Alpha 6, and $20 \mathrm{~S}$ mean fluorescence intensity (MFI) in cytoplasmic bodies using the Surface Finder function of the Imaris software package (Bitplane) and the data was plotted in Prism (Graphpad Software Inc) for statistical analysis. 


\section{Forster resonance energy transfer (FRET)} Immunofluorescent acceptor photobleaching in fixed cells Cells stably expressing HA-rhTRIM5 $\alpha$ [39] were seeded on coverslips at a subconfluent density. Coverslips were fixed with $3.7 \%$ formaldehyde (Polysciences) in $0.1 \mathrm{M}$ PIPES [piperazine- $N, N^{\prime}$-bis(2-ethanesulfonic acid)], $\mathrm{pH}$ 6.8. Cells were immunostained with a rabbit anti-HA primary antibody (Sigma) and mouse anti-PSMC2 or rabbit anti-20S primary antibodies (Enzo Life Sciences). Primary anti-HA antibody was secondarily labeled with Cy5-conjugated anti-rabbit antibody (Jackson ImmunoResearch), and proteasomal subunits were secondarily labeled with anti-mouse or anti-rabbit Alexa546 (Invitrogen). Cy5 fluorophore was bleached for total of two minutes every five seconds while fluorescence intensities were detected in the Alexa546 and Cy5 channels. Using SoftWoRx software, maximum intensities were analyzed over the course of the experiment for Alexa546 and Cy5 and graphed in Microsoft Excel.

\section{Fluorescent protein acceptor photobleaching in live cells}

FRET by acceptor photobleaching was performed as previously described [24]. Progressive acceptor photobleaching was performed as follows: 50 images were obtained at 10-second intervals for both donor (CFP: excitation 427/ 10, emission 473/30, $100 \mathrm{~ms}$ exposure) and acceptor (YFP: excitation 504/12, emission 542/27, 40 ms exposure), with a period of acceptor photobleaching (excitation 504/12) between each acquisition. The CFP/YFP fluorescence intensity of each cell in the field was quantified in Metamorph, and FRET efficiency was calculated from the CFP initial and final fluorescence values, according to $\mathrm{E}=1$ $\left(\mathrm{F}_{\text {prebleach }} / \mathrm{F}_{\text {postbleach }}\right)$.

Fluorescence imaging was performed with an inverted microscope equipped with a 1.49 numerical aperture objective, and a back-thinned CCD camera (iXon 887; Andor Technology, Belfast, Northern Ireland). Image acquisition and acceptor photobleaching was automated with custom software macros in Meta-Morph (Molecular Devices Corp., Downingtown, PA) that controlled motorized excitation/emission filter wheels (Sutter Instrument Co., Novato, CA) with filters for CFP/YFP/mCherry (Semrock, Rochester NY). The progressive photobleaching protocol was as follows: 100-ms acquisition of CFP image and 40-ms acquisition of YFP image, followed by 10-s exposure to YFP-selective photobleaching (504/12-nm excitation).

\section{E-FRET in live cells}

E-FRET was performed as previously described [44].

E-FRET was calculated according to:

$$
\mathrm{E}=\frac{\mathrm{I}_{\mathrm{DA}}-a\left(\mathrm{I}_{\mathrm{AA}}\right)-d\left(\mathrm{I}_{\mathrm{DD}}\right)}{\mathrm{I}_{\mathrm{DA}}-a\left(\mathrm{I}_{\mathrm{AA}}\right)+(\mathrm{G}-d)\left(\mathrm{I}_{\mathrm{DD}}\right)}
$$

where $I_{D D}$ is the intensity of fluorescence emission detected in the donor channel $(472 / 30 \mathrm{~nm})$ with $427 / 10$ $\mathrm{nm}$ excitation; $\mathrm{I}_{\mathrm{AA}}$ is the intensity of fluorescence emission detected in the acceptor channel with 542/27 nm emission and 504/12 nm excitation; $\mathrm{I}_{\mathrm{DA}}$ is the intensity of fluorescence emission detected in the "FRET" channel with $542 / 27 \mathrm{~nm}$ emission and 427/10 $\mathrm{nm}$ excitation; and $a$ and $d$ are cross-talk coefficients determined from acceptor-only or donor-only samples, respectively. We obtained a d value of 0.894 for CFP and a value of 0.108 for YFP. G is the ratio of the sensitized emission to the corresponding amount of donor recovery, which was 3.2.

\section{Additional material}

Additional file 1: TRIM5 $\alpha$ associates with proteasome subunit PSMC2 and 20S core particle in cells during immunofluorescence based FRET. (A) HeLa cells stably expressing HA-rhTRIM5 $\alpha$ were stained for HA using a primary anti-HA antibody, followed by a secondary antibody conjugated to Cy5 and endogenous PSMC2 using a mouse monoclonal antibody, followed by a secondary antibody conjugated to Alexa546. Z-stack images were collected and individual channel images were used to create the merged panels. (B) HeLa cells stably expressing HA-rhTRIM5 $\alpha$ were stained for HA using an antibody conjugated to Cy 5 and endogenous 205 core particle using a mouse monoclonal antibody conjugated to Alexa546. Z-stack images were collected and individual channel images were used to create the merged panels.

Additional file 2: YFP signal is progressively bleached during photoacceptor FRET. (A) HeLa cells were transfected with CFP-rhTRIM5 $\alpha$ and YFP-PSMC2. Images were collected throughout the acquisition process. Here we show CFP and YFP signal at the beginning (left) and at the end of the acquisition process (right). (B) HeLa cells were transfected with CFP-rhTRIM5 $\alpha$ and YFP empty vector. Images were collected throughout the acquisition process. Here we show CFP and YFP signal at the beginning (left) and at the end (right) of the acquisition process.

Additional file 3: Subcellular localization and characterization of proteasomal subunit antibodies using immunoflurescence. HeLa cells seeded on glass coverslips were fixed with $3.7 \%$ Formaldehyde (Methanol Free) in PIPES buffer (pH 6.8). Following fixation they were stained with various antibodies to proteasome subunits and imaged using a DeltaVision deconvolution microscope.

\section{Abbreviations}

rhTRIM5a: Rhesus macaque TRIM5a; RT: Reverse transcription; FRET: Forster resonance energy transfer.

\section{Acknowledgements}

This work was supported by National Institutes of Health grant K22 Al078757 and RO1 Al093258 to E. M.C., and SNF 3100AO-128655 and RO1 A/59159 to J.L.

We thank Dr. Shigeo Murata for providing plasmids expressing FLAG tagged proteasomal subunits and Dr. Ajay Rana for providing FLAG tagged MLK3 used in this study

\section{Author details}

'Department of Microbiology and Immunology, Stritch School of Medicine, Loyola University Chicago, Maywood IL, USA. ${ }^{2}$ Department of Microbiology and Molecular Medicine, University of Geneva, Geneva, Switzerland. ${ }^{3}$ Department of Cell and Molecular Physiology, Stritch School of Medicine, Loyola University Chicago, Maywood IL, USA. 


\section{Authors' contributions}

ZL designed, performed experiments, and wrote the manuscript. SH designed and performed experiments. SS designed and performed experiments. JR performed experiments. JS performed experiments. SLR designed experiments. JL designed experiments. EMC designed experiments and wrote the manuscript. All authors read and approved the final manuscript.

\section{Competing interests}

The authors declare that they have no competing interests.

Received: 31 August 2011 Accepted: 12 November 2011 Published: 12 November 2011

\section{References}

1. Lienlaf M, Hayashi F, Di Nunzio F, Tochio N, Kigawa T, Yokoyama S, Diaz Griffero F: Contribution of E3-Ubiquitin Ligase Activity to HIV-1 Restriction by TRIM5\{alpha\}rh: Structure of the RING Domain of TRIM5 \{alpha\}. J Virol 2011, 85:8725-8737.

2. Stremlau M, Owens CM, Perron MJ, Kiessling M, Autissier P, Sodroski J: The cytoplasmic body component TRIM5alpha restricts HIV-1 infection in Old World monkeys. Nature 2004, 427:848-853.

3. Reymond A, Meroni G, Fantozzi A, Merla G, Cairo S, Luzi L, Riganelli D, Zanaria E, Messali S, Cainarca S, et al: The tripartite motif family identifies cell compartments. Embo J 2001, 20:2140-2151.

4. Sayah DM, Sokolskaja E, Berthoux L, Luban J: Cyclophilin A retrotransposition into TRIM5 explains owl monkey resistance to HIV-1. Nature 2004, 430:569-573.

5. Nisole S, Lynch C, Stoye JP, Yap MW: A Trim5-cyclophilin A fusion protein found in owl monkey kidney cells can restrict HIV-1. Proc Natl Acad Sci USA 2004, 101:13324-13328.

6. Sebastian S, Luban J: TRIM5alpha selectively binds a restriction-sensitive retroviral capsid. Retrovirology 2005, 2:40.

7. Stremlau M, Perron M, Lee M, Li Y, Song B, Javanbakht H, Diaz-Griffero F, Anderson DJ, Sundquist WI, Sodroski J: Specific recognition and accelerated uncoating of retroviral capsids by the TRIM5\{alpha\} restriction factor. Proc Natl Acad Sci USA 2006.

8. Wu X, Anderson JL, Campbell EM, Joseph AM, Hope TJ: Proteasome inhibitors uncouple rhesus TRIM5alpha restriction of HIV-1 reverse transcription and infection. Proc Natl Acad Sci USA 2006, 103:7465-7470.

9. Anderson $\mathrm{J}$, Campbell EM, Wu X, Vandegraaff N, Engelman A, Hope TJ: Proteasome inhibition reveals that a functional preintegration complex intermediate can be generated during restriction by diverse TRIM5 proteins. J Virol 2006, 80:9754-9760.

10. Diaz-Griffero F, Kar A, Perron M, Xiang SH, Javanbakht H, Li X, Sodroski J: Modulation of Retroviral Restriction and Proteasome Inhibitor-resistant Turnover by Changes in the TRIM5\{alpha\} B-box 2 Domain. J Virol 2007, 81:10362-10378

11. Campbell EM, Perez O, Anderson JL, Hope TJ: Visualization of a proteasome-independent intermediate during restriction of HIV-1 by rhesus TRIM5alpha. J Cell Biol 2008, 180:549-561.

12. Rold CJ, Aiken C: Proteasomal degradation of TRIM5alpha during retrovirus restriction. PLoS Pathog 2008, 4:e1000074.

13. Pertel T, Hausmann S, Morger D, Zuger S, Guerra J, Lascano J, Reinhard C, Santoni FA, Uchil PD, Chatel L, et al: TRIM5 is an innate immune sensor for the retrovirus capsid lattice. Nature 2011, 472:361-365.

14. Murata S, Yashiroda H, Tanaka K: Molecular mechanisms of proteasome assembly. Nat Rev Mol Cell Biol 2009, 10:104-115.

15. Geetha T, Seibenhener ML, Chen L, Madura K, Wooten MW: p62 serves as a shuttling factor for TrkA interaction with the proteasome. Biochem Biophys Res Commun 2008, 374:33-37.

16. Murata S: Multiple chaperone-assisted formation of mammalian $20 \mathrm{~S}$ proteasomes. IUBMB Life 2006, 58:344-348.

17. Thylur RP, Senthivinayagam S, Campbell EM, Rangasamy V, Thorenoor N, Sondarva G, Mehrotra S, Mishra P, Zook E, Le PT, et al: Mixed Lineage Kinase 3 modulates \{beta\}-catenin signaling in cancer cells. J Bio/ Chem 2011.

18. Campbell EM, Dodding MP, Yap MW, Wu X, Gallois-Montbrun S, Malim MH, Stoye JP, Hope TJ: TRIM5 alpha cytoplasmic bodies are highly dynamic structures. Mol Biol Cell 2007 , 18.2102-2111.

19. Wojcik C, DeMartino GN: Intracellular localization of proteasomes. Int $J$ Biochem Cell Biol 2003, 35:579-589.
20. Klare N, Seeger M, Janek K, Jungblut PR, Dahlmann B: Intermediate-type 20 S proteasomes in HeLa cells: "asymmetric" subunit composition, diversity and adaptation. J Mol Biol 2007, 373:1-10.

21. Dahlmann B, Ruppert T, Kuehn L, Merforth S, Kloetzel PM: Different proteasome subtypes in a single tissue exhibit different enzymatic properties. J Mol Biol 2000, 303:643-653.

22. Luciani A, Villella VR, Esposito S, Brunetti-Pierri N, Medina D, Settembre C, Gavina M, Pulze L, Giardino I, Pettoello-Mantovani M, et al: Defective CFTR induces aggresome formation and lung inflammation in cystic fibrosis through ROS-mediated autophagy inhibition. Nat Cell Biol 2010, 12:863-875.

23. Hou Z, Kelly EM, Robia SL: Phosphomimetic mutations increase phospholamban oligomerization and alter the structure of its regulatory complex. J Biol Chem 2008, 283:28996-29003.

24. Kelly EM, Hou Z, Bossuyt J, Bers DM, Robia SL: Phospholamban oligomerization, quaternary structure, and sarco(endo)plasmic reticulum calcium ATPase binding measured by fluorescence resonance energy transfer in living cells. J Biol Chem 2008, 283:12202-12211.

25. O'Connor C, Pertel T, Gray S, Robia SL, Bakowska JC, Luban J, Campbell EM: p62/sequestosome- 1 associates with and sustains the expression of retroviral restriction factor TRIM5alpha. J Virol 2010, 84:5997-6006.

26. Takalkar AM, Klibanov AL, Rychak JJ, Lindner JR, Ley K: Binding and detachment dynamics of microbubbles targeted to P-selectin under controlled shear flow. J Control Release 2004, 96:473-482

27. Kenworthy AK: Imaging protein-protein interactions using fluorescence resonance energy transfer microscopy. Methods 2001, 24:289-296.

28. Ganser-Pornillos B, Chandrasekaran V, Pornillos O, Sodroski J, Sundquist WI, Yeager M: Hexagonal assembly of a restricting TRIM5alpha protein. Proc Natl Acad Sci USA 2010.

29. Ganser-Pornillos BK, Chandrasekaran V, Pornillos O, Sodroski JG, Sundquist WI, Yeager M: Hexagonal assembly of a restricting TRIM5alpha protein. Proc Natl Acad Sci USA 2011, 108:534-539.

30. McDonald D, Vodicka MA, Lucero G, Svitkina TM, Borisy GG, Emerman M, Hope TJ: Visualization of the intracellular behavior of HIV in living cells. J Cell Biol 2002, 159:441-452.

31. Diaz-Griffero F, Li X, Javanbakht H, Song B, Welikala S, Stremlau M, Sodroski J: Rapid turnover and polyubiquitylation of the retroviral restriction factor TRIM5. Virology 2006, 349:300-315.

32. Yamauchi K, Wada K, Tanji K, Tanaka M, Kamitani T: Ubiquitination of E3 ubiquitin ligase TRIM5 alpha and its potential role. FEBS J 2008, 275:1540-1555.

33. Rosenqvist $E$, Jossang T, Feder J: Thermal properties of human IgG. Mol Immunol 1987, 24:495-501.

34. Terry BR, Matthews EK, Haseloff J: Molecular characterisation of recombinant green fluorescent protein by fluorescence correlation microscopy. Biochem Biophys Res Commun 1995, 217:21-27.

35. Perez-Caballero D, Hatziioannou T, Zhang F, Cowan S, Bieniasz PD: Restriction of Human Immunodeficiency Virus Type 1 by TRIM-CypA Occurs with Rapid Kinetics and Independently of Cytoplasmic Bodies, Ubiquitin, and Proteasome Activity. J Virol 2005, 79:15567-15572.

36. Song B, Diaz-Griffero F, Park DH, Rogers T, Stremlau M, Sodroski J: TRIM5alpha association with cytoplasmic bodies is not required for antiretroviral activity. Virology 2005, 343:201-211.

37. Gyuris J, Golemis E, Chertkov H, Brent R: Cdi1, a human G1 and S phase protein phosphatase that associates with Cdk2. Cell 1993, 75:791-803.

38. Sastri J, O'Connor C, C D, M M, P P, Diaz-Griffero F, Campbell EM: Identification of residues within the L2 region of TRIM5a that are required for retroviral restriction and cytoplasmic body localization. Virology 2010, 405:259-266

39. O'Connor C, Pertel T, Gray S, Robia SL, Bakowska JC, Luban J, Campbell EM: p62/Sequestosome 1 associates with and sustains the expression of the retroviral restriction factor TRIM5\{alpha\}. J Virol 2010.

40. Hirano Y, Hayashi H, lemura S, Hendil KB, Niwa S, Kishimoto T, Kasahara M, Natsume T, Tanaka K, Murata S: Cooperation of multiple chaperones required for the assembly of mammalian $20 \mathrm{~S}$ proteasomes. $\mathrm{Mol} \mathrm{Cell}$ 2006, 24:977-984.

41. Durocher $Y$, Perret S, Kamen A: High-level and high-throughput recombinant protein production by transient transfection of suspensiongrowing human 293-EBNA1 cells. Nucleic Acids Res 2002, 30:E9.

42. Campbell EM, Nunez R, Hope TJ: Disruption of the actin cytoskeleton can complement the ability of Nef to enhance human immunodeficiency virus type 1 infectivity. J Virol 2004, 78:5745-5755. 
43. Sastri J, O'Connor C, Danielson CM, McRaven M, Perez P, Diaz-Griffero F, Campbell EM: Identification of residues within the $\mathrm{L} 2$ region of rhesus TRIM5alpha that are required for retroviral restriction and cytoplasmic body localization. Virology 2010, 405:259-266.

44. Zal T, Gascoigne NR: Photobleaching-corrected FRET efficiency imaging of live cells. Biophys J 2004, 86:3923-3939.

doi:10.1186/1742-4690-8-93

Cite this article as: Lukic et al.: TRIM $5 \alpha$ associates with proteasomal subunits in cells while in complex with HIV-1 virions. Retrovirology 2011 8:93.

Submit your next manuscript to BioMed Central and take full advantage of:

- Convenient online submission

- Thorough peer review

- No space constraints or color figure charges

- Immediate publication on acceptance

- Inclusion in PubMed, CAS, Scopus and Google Scholar

- Research which is freely available for redistribution 